\title{
AN EXACT SEQUENCE FOR SUBMERSIONS
}

\author{
BY J. WOLFGANG SMITH ${ }^{1}$
}

Communicated by S. Smale, October 10, 1967

1. Introduction. Let $X$ and $B$ denote topological spaces. By a topological submersion $\pi: X \rightarrow B$, we understand a continuous map of $X$ onto $B$ which admits local cross-sections. With such a map one can associate an exact homology sequence

$$
\cdots \rightarrow H_{q}(X) \rightarrow H_{q}(B) \rightarrow H_{q-1}^{r}(X) \rightarrow H_{q-1}(X) \rightarrow \cdots
$$

in which the terms $H_{q}(X), H_{q}(B)$ are singular integral homology groups and the terms $H_{a}^{r}(X)$ certain "residual homology groups" to be defined. We shall examine two special cases for which the residual groups can be identified with ordinary homology groups. The first result is as follows.

THEOREM 1. When $\pi$ is the projection of an oriented $k$-sphere bundle, there exist canonical isomorphisms $H_{a}^{r}(X) \approx H_{q-k}(B)$ by which (1.1) is reduced to the Thom-Gysin sequence.

As our second example we consider the case where $\pi$ is a differentiable submersion (in the usual sense) of codimension 1. Thus we assume that $X, B$ are $C^{\infty}$-manifolds of dimensions $n+1$ and $n$, respectively, and that $\pi$ is a regular differentiable map. Such a submersion is orientable if the induced line element field on $X$ is orientable, and $\pi$ will be called simple if $\pi^{-1}(b)$ is connected for all $b \in B$.

THEOREM 2. When $\pi$ is a simple oriented submersion of codimension 1 , there exist canonical isomorphisms $H_{a}^{r}(X) \approx H_{q-1}(U)$, where $U$ denotes the set of all $b \in B$ such that $\pi^{-1}(b)$ is compact. Thus (1.1) reduces to an exact sequence

$$
\cdots \rightarrow B_{q}(X) \rightarrow H_{q}(B) \rightarrow H_{q-2}(U) \rightarrow H_{q-1}(X) \rightarrow \cdots .
$$

Among the consequences which ensue from this result, we mention the following

CoROLLARY 1. Let $t^{2} \pi: E^{3} \rightarrow B$ be a simple submersion of codimension 1. Then $B$ must be $S^{2}$ or $E^{2}$, depending on whether there does or does not exist a point $b \in B$ such that $\pi^{-1}(b)$ is compact.

Corollary 2. If $\pi: E^{n+1} \rightarrow S^{n}$ is a simple submersion, then $n=2$.

1 Supported by National Science Foundation Grant GP-6648.

${ }^{2} E^{n}$ will denote Euclidean $n$-space and $S^{n}$ the $n$-sphere. 
(For $n=2$ such a submersion can be obtained from the Hopf fibration $S^{3} \rightarrow S^{2}$.)

2. The exact sequence. Given a topological submersion $\pi: X \rightarrow B$, let $C^{r}(X)$ denote the kernel of the induced homomorphism $\pi_{\sharp}: C(X) \rightarrow C(B)$ connecting the singular integral chain complexes, and let $C^{e}(X)$ denote the quotient of $C(X)$ by $C^{r}(X)$. One has thus a short exact sequence

$$
0 \rightarrow C^{r}(X) \stackrel{\alpha}{\rightarrow} C(X) \stackrel{\beta}{\rightarrow} C^{e}(X) \rightarrow 0,
$$

and this gives an exact sequence

$$
\cdots \rightarrow H_{q}^{r}(X) \stackrel{\alpha_{*}}{\rightarrow} H_{q}(X) \stackrel{\beta_{*}}{\rightarrow} H_{q}^{0}(X) \stackrel{\gamma}{\rightarrow} H_{q-1}^{r}(X) \rightarrow \cdots
$$

connecting the corresponding homology groups. One also obtains a chain monomorphism $\pi^{e}: C^{e}(X) \rightarrow C(B)$ such that $\pi^{e} \beta=\pi_{\sharp t}$. We will prove

LEMMA A. $\pi^{e}$ induces isomorphisms $\pi_{*}^{e}: H_{q}^{e}(X) \approx H_{q}(B)$ for all $q$.

Let $z \in C_{q}^{e}(X)$ be a cycle such that $\pi^{e}(z)=\partial c$ for some $c \in C_{q+1}(B)$. The existence of local cross-sections $s: B \rightarrow X$ implies that the barycentric subdivision $S d^{n} c$ admits a lifting $\bar{c} \in C_{q+1}(X)$ for $n$ sufficiently large. Thus we have $\pi_{*}(\bar{c})=S d^{n} c$, and consequently $\pi^{e} \partial \beta(\bar{c})=\pi^{e} S d^{n} z$. Since $\pi^{e}$ is injective, this gives $z \sim 0$, proving that $\pi_{*}^{e}$ is injective. Surjectivity follows by a similar argument.

This result permits us to rewrite (2.1) as an exact sequence

$$
\cdots \rightarrow H_{q}^{r}(X) \stackrel{\alpha_{*}}{\rightarrow} H_{q}(X) \stackrel{\pi_{*}}{\rightarrow} H_{q}(B) \stackrel{\delta}{\rightarrow} H_{q-1}^{r}(X) \rightarrow \cdots
$$

where $\delta=\gamma\left(\pi_{*}^{e}\right)^{-1}$.

3. Sphere bundles. Now let $\pi: X \rightarrow B$ be the projection of an oriented $k$-sphere bundle $\xi$. Moreover, we suppose that $\xi$ is imbedded in a vector bundle $p: E \rightarrow B$, so that $X$ may be identified with a subspace $\dot{E} \subset E$ (space of "unit vectors"). We propose to construct homomorphisms $\phi: H_{q-1}^{r}(\dot{E}) \rightarrow H_{q}(E, \dot{E})$ making the following diagram commute,

$$
\cdots \rightarrow H_{q}(\dot{E}) \stackrel{i_{*}}{\rightarrow} H_{q}(E) \stackrel{j_{*}}{\rightarrow} H_{q}(E, \dot{E}) \stackrel{\partial}{\rightarrow} H_{q-1}(\dot{E}) \rightarrow \cdots
$$


where $i_{*}, j_{*}$ are induced by inclusion maps and $\partial$ is the boundary operator. Since the horizontal lines in (3.1) are exact, it will follow by the 5-lemma that $\phi$ is an isomorphism.

Let $s: B \rightarrow E$ denote the zero cross-section, and $\tau: C(E) \rightarrow C(E)$ the chain homomorphism induced by $s p$. There now exists an obvious chain homotopy $D: 1 \simeq \tau$. More precisely, for $q \geqq 0$ and $\sigma: \Delta_{q} \rightarrow E$ a singular simplex, let $P_{\sigma}: \Delta_{q} \times I \rightarrow E$ denote the singular prism defined by $P_{\sigma}(x, t)=(1-t) \sigma(x)$. By the usual construction this gives homomorphisms $D_{q}: C_{q}(E) \rightarrow C_{q+1}(E)$ such that

$$
\partial D+D \partial=\tau-1 .
$$

Consider $d: C^{r}(\dot{E}) \rightarrow C(E, \dot{E})$ defined by $d=j D \alpha$. Since $C^{r}(\dot{E}) \subset \operatorname{ker} \tau$, (3.2) gives $\partial d=-d \partial$, so that $d$ is an "antihomomorphism" of degree +1 . It consequently induces homomorphisms $\phi: H_{q-1}^{r}(\dot{E}) \rightarrow H_{q}(E, \dot{E})$.

To establish commutativity of (3.1), one first observes that $\pi_{*}^{e} \beta_{*}=p_{*} i_{*}$, leaving

$$
\begin{aligned}
j_{*} p_{*}^{-1} & =\phi \delta, \\
\partial \phi & =\alpha_{*}
\end{aligned}
$$

to be verified. Consider an element $h \in H_{q}(B)$. Since $\pi=p \mid \dot{E}$ admits local cross-sections, one can choose a representative cycle $z=\sum a_{i} \sigma_{i}$ of $h$ such that each singular simplex $\sigma_{i}$ admits a lifting $\bar{\sigma}_{i}$ to $\dot{E}$. Setting $\bar{z}=\sum a_{i} \bar{\sigma}_{i}$ one obtains a "residual" cycle $\partial \bar{z} \in C_{a-1}^{r}(\dot{E})$ representing $\delta(h)$. Mapping $\partial \bar{z}$ to $C_{q}(E, \dot{E})$ by $d$ gives a cycle $z^{\prime}$ representing $\phi \delta(h)$. One sees by a short calculation that $z^{\prime} \sim s_{\sharp}(z)$ (where $s_{\sharp}: C(B)$ $\rightarrow C(E, \dot{E})$ denotes the chain map induced by $s)$, and this proves (3.3). The verification of (3.4) is also easy, and one thus obtains

Lemma B. There exist isomorphisms $\phi: H_{q-1}^{r}(\dot{E}) \approx H_{q}(E, \dot{E})$ satisfying (3.3) and (3.4).

Setting $\Omega=\Phi \phi$, where $\Phi: H_{q}(E, \dot{E}) \rightarrow H_{q-k-1}(B)$ denotes the Thom isomorphism, one has

$$
\begin{aligned}
& \ldots \rightarrow B_{q}(E) \stackrel{\pi_{*}^{*}}{\rightarrow} H_{q}(B) \stackrel{\Psi}{\rightarrow} H_{q-k-1}(B) \stackrel{\rho}{\rightarrow} H_{q-1}(E) \rightarrow \cdots \\
& =\uparrow=\uparrow \quad \uparrow \Omega \quad \uparrow= \\
& \cdots \rightarrow H_{q}(E) \stackrel{\pi_{*}}{\rightarrow} H_{q}(B) \stackrel{\delta}{\rightarrow} B_{q-1}^{r}(E) \stackrel{\alpha_{*}}{\rightarrow} H_{q-1}(E) \rightarrow \cdots
\end{aligned}
$$

where $\Psi=\Phi j_{*} p_{*}^{-1}$ and $\rho=\partial \Phi^{-1}$. Conditions (3.3) and (3.4) imply commutativity. Moreover, the top line gives the Thom-Gysin sequence $^{8}$ of $\xi$.

See Spanier [21, p. 260]. 
4. Submersions of codimension 1. Let $\pi: X \rightarrow B$ denote a simple submersion of codimension 1 . We set $\dot{E}=\pi^{-1}(U)$ and consider the exact sequence

$$
\cdots \rightarrow H_{q}^{r}(E) \rightarrow H_{q}^{r}(X) \rightarrow H_{q}^{r}(X, E) \rightarrow H_{q-1}^{r}(E) \rightarrow \cdots
$$

connecting the residual homology groups. As a special case of a theorem established in [1], we obtain

Lemma C. $H_{a}^{r}(X, \dot{E})=0$ for all $q$.

The idea of the proof is this: Every residual cycle $z \in C_{a}^{+}(x)$ can be written in the form $z=\sum a_{i}\left(\sigma_{i}-\sigma_{i}^{\prime}\right)$ with $\pi \sigma_{i}=\pi \sigma_{i}^{\prime}$. One can now define singular prisms $P_{i}: \Delta_{q} \times I \rightarrow X$ connecting $\sigma_{i}$ and $\sigma_{i}^{\prime}$ such that $\pi P_{i}(x, t)=\pi \sigma_{i}(x)$ for all $(x, t)$. A suitably defined "triangulation" of $P_{i}$ will yield residual chains $D_{i} \in C_{a+1}^{r}(X)$. We can define chains $D \partial_{i}$ by the formula $\partial D_{i}+D \partial_{i}=\sigma_{i}-\sigma_{i}^{\prime}$ and thus obtain $z \sim \sum a_{i} D \partial_{i}$. It can be shown that $\sum a_{i} D \partial_{i} \sim 0 \bmod \dot{E}$.

By Lemma $C$ and exactness of (4.1) we have isomorphisms $H_{a}^{r}(\dot{E}) \approx H_{a}^{r}(X)$. On the other hand, $\pi \mid \dot{E}$ is the projection of a 1 -sphere bundle over $U$. Hence $H_{a}^{r}(\dot{E}) \approx H_{q-1}(U)$ by Theorem 1 , and this establishes Theorem 2.

\section{REFERENCES}

1. B. C. Chang and J. W. Smith, Singular homology of topological objects (to appear).

2. E. H. Spanier, Algebraic topology, McGraw-Hill, New York, 1966.

Oregon State University 